\title{
Compacting and correcting Trinity and Oases RNA-Seq de novo assemblies
}

\author{
Cédric Cabau ${ }^{\text {Corresp., }}{ }^{1}$, Frédéric Escudié ${ }^{2}$, Anis Djari ${ }^{3}$, Yann Guiguen ${ }^{4}$, Julien Bobe ${ }^{4}{ }^{\text {, Christophe Klopp }}{ }^{2}$ \\ 1 SIGENAE, GenPhySE, Université de Toulouse, INRA, INPT, ENV, Castanet Tolosan, France \\ 2 Plate-forme bio-informatique Genotoul, Mathématiques et Informatique Appliquées de Toulouse, INRA, Castanet Tolosan, France \\ 3 Laboratoire Génomique et Biotechnologie du Fruit, UMR990 INRA/INP-ENSAT, Auzeville, France \\ 4 UR1037 Fish Physiology and Genomics, INRA, Rennes, France \\ Corresponding Author: Cédric Cabau \\ Email address: Cedric.Cabau@inra.fr
}

\section{Background}

De novo transcriptome assembly of short reads is now a common step in expression analysis of organisms lacking a reference genome sequence. Several software packages are available to perform this task. Even if their results are of good quality it is still possible to improve them in several ways including redundancy reduction or error correction. Trinity and Oases are two commonly used de novo transcriptome assemblers. The contig sets they produce are of good quality. Still, their compaction (number of contigs needed to represent the transcriptome) and their quality (chimera and nucleotide error rates) can be improved.

\section{Results}

We built a de novo RNA-Seq Assembly Pipeline (DRAP) which wraps these two assemblers (Trinity and Oases) in order to improve their results regarding the above-mentioned criteria. DRAP reduces from 1.3 to 15 fold the number of resulting contigs of the assemblies depending on the read set and the assembler used. This article presents seven assembly comparisons showing in some cases drastic improvements when using DRAP. DRAP does not significantly impair assembly quality metrics such are read realignment rate or protein reconstruction counts.

\section{Conclusion}

Transcriptome assembly is a challenging computational task even if good solutions are already available to end-users, these solutions can still be improved while conserving the overall representation and quality of the assembly. The de novo RNA-Seq Assembly Pipeline (DRAP) is an easy to use software package to produce compact and corrected transcript set. DRAP is free, open-source and available under GPL V3 license at http://www.sigenae.org/drap . 


\section{Title}

2 Compacting and correcting Trinity and Oases RNA-Seq de novo assemblies

\section{Authors}

4 Cédric Cabau $^{1 *}$, Frédéric Escudié2 ${ }^{*}$, Anis Djari ${ }^{3}$, Yann Guiguen ${ }^{4}$, Julien Bobe $^{4}$, Christophe

$5 \quad \mathrm{Klopp}^{2}$

$6 *$ equal contribution

\section{Affiliations}

$8{ }^{1}$ SIGENAE, GenPhySE, Université de Toulouse, INRA, INPT, ENVT, Castanet Tolosan, France

9 2 Plate-forme bio-informatique Genotoul, Mathématiques et Informatique Appliquées de

10 Toulouse, INRA, Castanet-Tolosan, France

$11{ }^{3}$ Laboratoire Génomique et Biotechnologie du Fruit, UMR990 INRA/INP-ENSAT, 24, Chemin

12 de Borde Rouge - Auzeville, France

$13{ }^{4}$ INRA, UR1037 Fish Physiology and Genomics, F-35000 Rennes, France

\section{Corresponding Author}

15 Cédric Cabau

16 Cedric.Cabau@inra.fr 


\section{Abstract}

19 Background

20 De novo transcriptome assembly of short reads is now a common step in expression analysis of

21 organisms lacking a reference genome sequence. Several software packages are available to

22 perform this task. Even if their results are of good quality it is still possible to improve them in

23 several ways including redundancy reduction or error correction. Trinity and Oases are two

24 commonly used de novo transcriptome assemblers. The contig sets they produce are of good

25 quality. Still, their compaction (number of contigs needed to represent the transcriptome) and

26 their quality (chimera and nucleotide error rates) can be improved.

27 Results

28 We built a de novo RNA-Seq Assembly Pipeline (DRAP) which wraps these two assemblers

29 (Trinity and Oases) in order to improve their results regarding the above-mentioned criteria.

30 DRAP reduces from 1.3 to 15 fold the number of resulting contigs of the assemblies depending

31 on the read set and the assembler used. This article presents seven assembly comparisons

32 showing in some cases drastic improvements when using DRAP. DRAP does not significantly

33 impair assembly quality metrics such are read realignment rate or protein reconstruction counts.

34 Conclusion

35 Transcriptome assembly is a challenging computational task even if good solutions are already

36 available to end-users, these solutions can still be improved while conserving the overall

37 representation and quality of the assembly. The de novo RNA-Seq Assembly Pipeline (DRAP) is

38 an easy to use software package to produce compact and corrected transcript set. DRAP is free,

39 open-source and available under GPL V3 license at http://www.sigenae.org/drap. 
41 Keywords: RNA-Seq, de novo assembly, compaction, correction, quality assessment

\section{Background}

Second generation sequencing platforms have enabled the production of large amounts of transcriptomic data permitting to analyze gene expression for a large variety of species and conditions. For species lacking a reference genome sequence, the now classical processing pipeline includes a de novo transcriptome assembly step. Assembling an accurate transcriptome reference is difficult because of the raw data variability. This variability comes from different factors: including 1 . The variability of gene expression levels ranging usually between one and millions of copies, 2 . The biology of mRNA synthesis which goes through an early stage of premRNA still containing introns and a late state in which mRNA can be decayed, 3 . The synthesis

53 from pre-mRNA of numerous alternative transcripts, 4. Potential sample contaminations, 5.

54 Sequencing quality biases, 6. Most of the genome can be expressed in low abundance depending on the biological condition as presented by Djebali et al.[1] in the results of the ENCODE 56 project.

58 Today there is no unique best solution to these RNA-Seq assembly problems but several software packages have been proven to generate contig sets comprising most of the expressed transcripts correctly reconstructed. Trinity [2] and Oases [3] are good examples. The assembled

61 contig sets produced by these packages often contain multiple copies of complete or partial

62 transcripts and also chimeras. Chimeras are structural anomalies of a unique transcript (self-

63 chimeras) or multiple transcripts (multi-transcripts chimeras). They are called "cis" if the 
64 transcripts are in the same direction and "trans" if they are in opposite directions. Natural

65 chimeric transcripts exist in some cancer tissues but are rare [4]. Yang and Smith [5] have

66 shown the tendency of de novo transcriptome assemblers to produce self-chimeric contigs. The

67 prevalence of the phenomenon depends on the assembly parameters. Multi-transcript chimeras

68 distort contig annotation. The functions of the transcripts merged in the same contig can be very

69 different and therefore the often-unique annotation given to such a chimeric contig does not

70 reflect its content. Assemblies include also contigs corresponding to transcription or sequencing

71 noise a phenomenon often referred as illegitimate transcription [6]. These contigs have often low

72 coverage and are not found in the different replicates of the same condition.

74 Some contigs contain local biological variations or sequencing errors such as substitutions,

75 insertions or deletions. These variations and errors can deeply impact the read alignment rate,

76 create frameshifts which hinder annotation, limit the efficacy of primer design and generate false

77 variations. Assemblies contain also polyA/T tails, which are posttranscriptional marks. They are

78 usually removed before publication. For all these reasons contig sets usually need error

79 correction.

81 Trinity and Oases have different algorithms, which give them advantages or disadvantages

82 depending on gene expression levels. The main difference comes from their assembly strategy.

83 Trinity chains a greedy algorithm with a de Bruijn graph one and Oases uses multiple de Bruijn

84 graphs with different $k$-mers. The first step of Trinity is very effective in assembling parts of

85 highly expressed transcripts which will be connected at the second step. As shown by Yann

86 Surget-Groba and Juan I. Montoya-Burgos [7], the Oases multi- $k$ assembly approach is able to 
87 build contigs corresponding to transcripts with very low to very high expression levels. However

88 highly expressed genes with multiple transcripts will generate very complex graphs mainly

89 because of the presence of variations or sequencing errors which will form new paths possibly

90 considered as valid by the assembler and produce numerous erroneous contigs. No assembler is

91 producing the best contig set in all situations. Bio-informaticians and biologists therefore use

92 different strategies to maximize the reference contig set quality [8][9][10][11]. The simplest

93 approach is to produce a reference set per software package or parameter set, to compare their

94 metrics and choose the best one. It is also possible to merge different results and filter them.

95

Assemblies can be compared on different criteria. The usual ones are simple contig metrics such as total count, total length, N50, average length.... Assembling equals summarizing (compressing the expression dimension) and therefore a good metric to check the summary quality is the proportion of reads mapped back to the contigs. As a large part of the transcripts correspond to mRNA, it is also possible to use as quality metric the number of correctly reconstructed proteins using a global reference as it is done by CEGMA [12] or BUSCO [13] or using a protein reference set from a phylogenetically closely related organism. Last, some software packages are also rating the contig set or the individual contigs using the above-mentioned criteria [14] or some other for example only related to the way reads map back to the contigs [15][16][17].

We have built a de novo RNA-Seq Assembly Pipeline (DRAP) in order to correct the following assembly problems: multiple copies of complete or partial transcripts, chimeras, lowly expressed

108 intergenic transcription, insertion and deletion generated by the assemblers and polyA tails. The

109 pipeline implementation is presented in the next section. The "results and discussion" section 
110 compares raw and DRAP assembly metrics for seven different datasets.

111

\section{Implementation}

113

114 DRAP is written in Perl, Python, and shell. The software is a set of three command-line tools

115 respectively called runDrap, runMeta and runAssessment. runDrap performs the assembly

116 including compaction and correction. It produces a contig set but also a HTML log report

117 presenting different assembly metrics. runAssessment compares different contig sets and gathers

118 the results in a global report. runMeta merges and compacts different contigs sets and should be

119 used for very large datasets for which memory or CPU requirements do not enable a unique

120 global assembly or for highly complex datasets. The modules chained by each tool are presented

121 in a graphical manner in Fig. 1, 2 and 3. Details on the compaction, correction and quality

122 assessment steps of the tools are described hereafter. All software versions, parameters and

123 corresponding default values are presented in Table S1.

124

125 Contig set compaction

126

127 Contig compaction removes redundant and lowly expressed contigs. Four different approaches

128 are used to compact contig sets. The first is only implemented for Oases assemblies and

129 corresponds to the sub-selection of only one contig per locus (NODE) produced by the

130 assembler. Oases resolves the connected component of the de Bruijn graph and for complex sub-

131 graphs generates several longest paths corresponding to different possible forms. These forms

132 have shown (https://sites.google.com/a/brown.edu/bioinformatics-in-biomed/velvet-and-oases- 
133 transcriptome) to correspond to subpart of the same transcript, which are usually included one in

134 another. Oases provides the locus (connected component of the assembly graph) of origin of

135 each contig as well as its length and depth. The Oasesv2.0.4BestTransChooser.py script sub-

136 selects the longest and most covered contig of a locus. The second compaction method removes

137 contigs included in longer ones. CD-HIT-EST [18] orders the contigs by length and removes all

138 the included ones given identity and coverage thresholds. The third method elongates the contigs

139 through a new assembly step. TGICL [19] performs this assembly in DRAP. The last approach

140 filters contigs using their length or the length of their longest ORF if users are only interested in

141 coding transcripts, and using read coverage according to the idea that lowly covered contigs

142 often correspond to noise. A last optional filter selects contigs using their TransRate quality

143 score when above the calculated threshold (--optimize parameter). By default, runDrap produces

144 eight contigs sets, four include only protein coding transcripts and four others contain all

145 transcripts. Each group comprises a contig set filtered for low coverage with respectively 1, 3, 5

146 and 10 fragments per kilobase per million (FPKM) thresholds.

147 Compaction favors assemblies having contigs with multiple ORFs. Because a unique ORF is

148 expected for contig annotation, DRAP splits multi-transcript chimera in mono-ORF contigs.

150 runMeta also performs a three step compaction of the contigs. The first is based on the contig

151 nucleotide content and uses CD-HIT-EST. The second run CD-HIT on the protein translation of

152 the longest ORF found by EMBOSS getorf. The third, in the same way as runDrap, filters

153 contigs using their length (global or longest ORF), their expression level and optionally their

154 TransRate score producing the eight result files described in the previous paragraph.

155 Contig set corrections 
157 Contig correction splits chimeras, removes duplicated parts, removes insertions, deletion and 158 polyA/T tails. DRAP corrects contigs in three ways. It first searches self-chimera and removes

159 them by splitting contigs in parts or removing duplicated chimeric elements. An in house script 160 aligns contigs on themselves using bl2seq and keeps only matches having an identity greater or 161 equal to $96 \%$. A contig is defined as a putative chimera if i) the longest self-match covers at least $16260 \%$ of the contig length or ii) the sum of partial non-overlapping self-matches covers at least

$16380 \%$ of its length. In the first case, the putative chimera is split at the start position of the

164 repeated block. In the second case, the contig is only a repetition of a short single block and is

165 therefore discarded. For the second correction step, DRAP searches substitutions, insertions and

166 deletions in the read realignment file. When found it corrects the consensus according to the

167 most represented allele at a given position. Low read coverage alignment areas are usually not

168 very informative therefore only positions having a minimum depth of 10 reads are corrected. The

169 manual assessment made on DRAP assemblies has shown that a second path of this algorithm

170 improves consensus correction. Part of the reads change alignment location after the first

171 correction. runDrap, consequently, runs this step twice.

172 The last correction script eases the publication of the contig set in TSA

173 (https://www.ncbi.nlm.nih.gov/genbank/tsa): NCBI transcript sequence assembly archive. TSA

174 stores the de novo assembled contig sets of over 1300 projects. In order to improve the data

175 quality, it performs several tests before accepting a new submission. These tests search for

176 different elements such as sequencing adapters or vectors, polyA or polyT and stretches of

177 unknown nucleotides $(\mathrm{N})$. The thresholds used by TSA are presented at

178 https://www.ncbi.nlm.nih.gov/genbank/tsaguide. DRAP performs the same searches on the 
179 contig set and corrects the contigs when needed.

180

181 Quality assessment

182

183 All three workflows create an HTML report. The report is a template including HighCharts

184 (http://www.highcharts.com) graphics and tables using JSON files as database. These files are

185 generated by the different processing steps. The report can therefore also be used to monitor

186 processing progression. Each graphic included in the report can be downloaded in PNG, GIF,

187 PDF or SVG. Some of the graphics can be zoomed in by mouse selecting the area to be enlarged.

188 The report tables can be sorted by clicking on the column headers and exported in CSV format.

189 For runDrap and runMeta, the reports present results of a single contig file.

190

191 runAssessment processes one or several contig files and one or several read files. It calculates

192 classical contig metrics, checks for chimeras, searches alignment discrepancies, produces read

193 and fragment alignment rates and assess completeness using an external global reference running

194 BUSCO. If provided, it aligns a set of proteins on the contigs to measure their overlap. Last, it

195 runs TransRate, a contig validation software using four alignment linked quality measures to

196 generate a global quality criterion for each contig and for the complete set. runAssessment does

197 not modify the contig set content but enables users to check and select the best candidate

198 between different assemblies.

199

200 Parallel processing and flow control

201 
202 DRAP runs on Unix machines or clusters. Different steps of the assembly or assessment process

203 are run in parallel mode, if the needed computer infrastructure is available. All modules have

204 been implemented to take advantage of an SGE compliant HPC environment. They can be

205 adapted to other schedulers through configuration file modification.

206 DRAP first creates a set of directories and shell command files and then launches these files in

207 the predefined order. The '--write' command line parameter forces DRAP to stop after the first 208 step. At this stage, the user can modify the command files for example to set parameters which

209 are not directly accessible from runDRAP, runMeta or runAssessment and then launch the

210 process with the '--run' command line option.

211 DRAP checks execution outputs at each processing step. If an error has occurred, it adds an error

212 file to the output directory indicating at which step of the processing it happened. After

213 correction, DRAP can be launched again and it will scan the result directory and restart after the

214 last error free step. The pipeline can easily be modified to accept other assemblers by rewriting

215 the corresponding wrapper using the input files and producing correctly named output files.

\section{Results and discussion}

218 DRAP has been tested on seven different datasets corresponding to five species. These datasets

219 are presented in Table 1 and include five real datasets (Arabidopsis thaliana: At, Bos taurus: $\mathrm{Bt}$, 220 Drosophila melanogaster: Dm, Danio rerio: Dr and Homo sapiens: Hs), one set comprising a

221 large number of diverse samples (Danio rerio multi samples: Dd) and one simulated dataset

222 (Danio rerio simulated: Ds). The simulated reads have been produced using rsem-simulate-reads

223 (version rsem-1.2.18)[20]. The theta0 value was calculated with the rsem-calculate-expression

224 program on read files from the Danio rerio pineal gland sample (SRR1048059). Table 1 also 
225 presents for each dataset: the number, length, type (paired or not) and strandedness of the reads,

226 the public accession number, the tissue and experimental condition of origin. The results

227 presented hereafter compare the metrics collected from Trinity, Oases, DRAP Trinity and DRAP

228 Oases assemblies of the six first datasets. The multi sample dataset has been used to compare a

229 strategy in which all reads of the different samples are gathered and processed as one dataset

230 (pooled) to a strategy in which the assemblies are performed by sample and the resulting contigs

231 joined afterwards (meta-assembly). The same assembly pipeline has been used in both strategies,

232 except the contig set merging step, which is specific to the meta-assembly strategy.

233

234 Summary Table 2 and Table 3 present the metrics collected for the six first datasets. Table 2

235 provides metrics related to compaction and correction as Table 3 includes validation metrics and

236 Table 4 collects all three metric types for pooled versus meta-assembly strategies.

238 Contig set compaction:

239

240 The improvement in compactness is measured by three criteria. The first is the number of

241 assembled contigs presented in Fig. 4. The differences between raw Oases and Trinity

242 assemblies and DRAP assemblies are very significant ranging from 1.3 fold to 15 fold. The

243 impact of DRAP on Oases assemblies (from 3.4 to 15 fold) is much more significant than on

244 Trinity assemblies (from 1.3 to 2,2 fold). Oases multi- $k$ assembly strategy generates a lot of

245 redundant contigs which are not removed at the internal Oases merge step. The second criterion

246 is the percentage of inclusions, i.e. contigs which are part of longer ones. Oases and Trinity

247 inclusion rate range respectively from 55 to $75 \%$ and from 2.3 to $5.5 \%$ (Table 2). Because of its 
248 inclusion removal step this rate is null for DRAP assemblies. The last compaction criteria

249 presented here is the total number of nucleotides in the contigs. The ratios between raw and

250 DRAP assembly sizes for Oases and Trinity range respectively from 3.4 to 14.8 fold and from

2511.1 and 2.6 fold (Table 2). All these metrics show that DRAP produces less contigs with less

252 redundancy resulting in an assembly with a much smaller total size.

253 Another metric that can be negatively correlated to compactness, but has to be taken into

254 account, is the number of multi-ORF contigs found in the assemblies. The ratios of multi-ORF

255 contigs found between raw and DRAP assemblies range from 11 and 116 folds (Table 2). DRAP

256 multi-transcript chimera splitting procedure improves significantly this criterion.

257 In order to check if the compaction step only selects one isoform per gene, we compared the

258 number of genes with several transcripts aligning on different contigs before and after DRAP. A

259 transcript is linked to a contig if its best blat hit has over $90 \%$ query identity and $90 \%$ query

260 coverage. The test has been performed on the Dr and the Ds datasets assembled with Oases and

261 Trinity. The number of alternative spliced isoforms decreases more, with or without DRAP, in

262 the Oases than in the Trinity assemblies (Table 5). This reduction is of $69 \%$ and $23 \%$ in the real

263 dataset (Dr) and $83 \%$ and $18 \%$ in the simulated dataset for Oases and Trinity respectively.

264 However, the spliced forms reduction does not impact the gene representation in the compacted

265 sets (Table 5). Remarkably, the gene representation is increased for the real dataset when

266 processed with DRAP Oases. This results from the different merging strategies used by Oases

267 and DRAP Oases. Using TGICL, DRAP is able, in some cases, to correctly merge gene parts

268 which have been generated by the Oases multi- $k$ assemblies and this more efficiently than the

269 build-in Oases merge procedure. 
271 Contig set corrections

272

273 DRAP corrects contigs in two ways: removing self-chimera and rectifying consensus

274 substitutions, insertions and deletions when the consensus does not represent the major allele at

275 the position in the read re-alignment file. Self-chimeras appear in Oases and Trinity contig sets at

276 rate ranging respectively from 0.11 to 1.39 and from 0.09 to $0.56 \%$. In DRAP, the corresponding

277 figures drop to 0.01 to 0.16 and 0.00 to $0.01 \%$. Concerning consensus correction only five

278 datasets can be taken into account i.e. At, Bt, Dm, Ds and Hs. Dr Oases assembly generates such

279 a large number of contigs and total length that it decreases significantly the average coverage and

280 therefore limits the number of positions for which the correction can be made. As shown in Fig.

2815 and Table S2, the Dr dataset is an outlier concerning this criteria. Regarding the five other

282 datasets raw versus DRAP correction rates range from 1.7 to 18.6 for insertions, 3.1 to 27.1 for

283 deletions and 2.7 to 14.1 for substitutions. DRAP correction steps lowers significantly the

284 number of positions for which the consensus does not correspond to the major allele found in the

285 alignment. In order to check the positive impact of the correction step, the Danio rerio reference

286 proteome has been aligned to the simulated dataset (Ds) contigs before and after correction.

$28794.5 \%$ of DRAP Oases contigs and 86.2\% of DRAP Trinity contigs which have been corrected,

288 have improved alignment scores (supplemental data S1 section "Contig set correction step

289 assessment").

290

291 Assembly quality assessment

292

293 The two previous parts have shown the beneficial impacts of DRAP on the assembly 
294 compactness and error rates but this should not impair quality metrics such as read and read pairs

295 alignment rates, number of ORFs, complete ORFs found in the contigs, number of proteins of the

296 known proteome mapped on the contigs or TransRate marks.

297 Read and read pair alignment rates differences between raw and DRAP assemblies are usually 298 very low, between 1 and 2\% and can sometimes be in favor of DRAP (Fig. 6) . In our test sets, 299 the difference is significant (7.5\%) for Dm when comparing Trinity to DRAP Trinity. This

300 comes from the removal by DRAP of a highly expressed transcript (Ensembl: FBtr0100888

301 mitochondrial large ribosomal RNA) because that does not fulfill the criteria of having at least

302 one 200 base pairs long ORF despite having over $11 \mathrm{M}$ reads aligned on the corresponding contig

303 in the Trinity assembly. DRAP Oases assembly was not impacted because it builds a longer

304 contig for this transcript with a long enough ORF to be selected in the additional part.

305 The reference proteome has been aligned on the contigs and matches with over $80 \%$ identity and

$30680 \%$ protein coverage have been counted (Fig. 7). These figures give a good overview of the

307 amount of well-reconstructed proteins in the contig sets. For all datasets except one (At) the

308 number of proteins are very close between raw and DRAP results. For this At dataset the

309 difference is of $12.2 \%$ for Oases and $13.2 \%$ for Trinity. This is due to the FPKM filtering step

310 performed by DRAP and the expression profile of this dataset that mixes different tissues (root,

311 shoot and flower) and conditions (full nutrition and starvation). Contigs corresponding to low

312 expression in one condition do not have sufficient overall expression to pass DRAP expression

313 filter threshold and are therefore eliminated from the final set. Mixed libraries can benefit from

314 the meta-assembly approach presented in the next section.

315 TransRate global scores (Fig. 8) are much higher for DRAP assemblies compared to raw ones.

316 This comes from the compaction performed by DRAP and the limited impact it has on the read 
317 alignment rate.

318 DRAP has limited negative effect on the assembly quality metrics, and sometimes even improves

319 some of them. Some cases in which multiple libraries are mixed with very distinct conditions can

320 affect the results and it is good practice to systematically compare raw and DRAP assemblies. It

321 is also to be noticed that Oases multi- $k$ strategy outperforms Trinity for all datasets regarding the

322 number of well-reconstructed proteins.

323

324 Pooled versus meta-assembly strategies

325

326 In the previous sections we compared results from raw and DRAP assemblies. This section

327 compares results from pooled versus meta-assembly strategies both using the DRAP assembly

328 pipeline (Table 4). Because of the read re-alignment filtering thresholds used in DRAP, we

329 expect different metrics between a pooled assembly and merged per sample assembly (meta-

330 assembly). DRAP includes the runMeta workflow, which performs this task.

331 Differences in compaction and correction are more important between Trinity and Oases than

332 between pooled versus meta-assembly. Pooled assemblies collect significantly worse results for

333 the number of reference proteins and number of read pairs aligned on the contigs. This comes

334 from the filtering strategy which eliminates low-expressed contigs of a given condition when

335 merging all the samples but will keep these contigs in a per sample assembly and meta-assembly

336 strategy. Therefore, we recommend using runMeta when the assembly input samples mix distinct

337 conditions with specific and variable expression patterns.

338

339 Assemblies fidelity check using simulated reads 
341 The simulation process links each read with its transcript of origin. With this information it is

342 possible to link contigs and transcripts. Here, the transcript-contig link was calculated using exon

343 content and order in both sets (method explained in Data S1). The results presented in Table 6

344 first shows that the assembly process loses between 15.76 and $19.97 \%$ of the exons compared to

345 the initial transcript set. This loss is close to $22 \%$ for all assemblies when the exon order is taken

346 into account. As shown in Fig. 9, this is mainly the case for transcripts with low read coverage.

347 The figures show once more that DRAP has a very limited negative impact on number of

348 retrieved exons in correct order.

349 Table 6 shows the number of contigs linked to more than one gene. DRAP compaction and ORF

350 splitting feature could have an antagonist impact for this criteria. But depending on the

351 assembler, the figures are in favor or not of DRAP.

352 Table 6 also presents the maximum number of genes linked to a single contig. These clusters

353 correspond to zink finger gene family members which have been assembled as single contig.

354 Between 92.3 and $93.7 \%$ of the clustered transcripts belong to this family. De novo assembly

355 tools are not able to distinguish transcript originating from different gene when the nucleotide

356 content is highly similar.

358 Conclusion

359 Different software packages are available to assemble de novo transcriptomes from short reads.

360 Trinity and Oases are commonly used packages which produce good quality references. DRAP

361 assembly pipeline is able to compact and correct contig sets with usually very low quality loss.

362 As no package out performs the others in all cases, producing different assemblies and 
363 comparing their metrics is a good general practice.

364

365 Abbreviations

366 RMP: reads per million

367 ORF: open reading frame

368 TSA: Transcript sequences archive

369 NCBI: National Center for Bio-Informatics

370 DRAP: De novo Rna-seq Assembly Pipeline

371 PNG, GIF, PDF or SVG: are image file formats.

372

373 References

1. Djebali S, Davis CA, Merkel A, Dobin A, Lassmann T, Mortazavi A, et al. 2012. Landscape of transcription in human cells. Nature 489:101-8.

2. Grabherr MG, Haas BJ, Yassour M, Levin JZ, Thompson DA, Amit I, et al. 2011. Full-length transcriptome assembly from RNA-Seq data without a reference genome. Nat. Biotechnol. 29:644-52.

3. Schulz MH, Zerbino DR, Vingron M, Birney E. 2012. Oases: robust de novo RNA-seq assembly across the dynamic range of expression levels. Bioinformatics 28:1086-92.

4. Frenkel-Morgenstern M, Gorohovski A, Lacroix V, Rogers M, Ibanez K, Boullosa C, et al. 2013. ChiTaRS: a database of human, mouse and fruit fly chimeric transcripts and RNAsequencing data. Nucleic Acids Res. 41:D142-51.

5. Yang Y, Smith SA. 2013. Optimizing de novo assembly of short-read RNA-seq data for phylogenomics. BMC Genomics 14:328.

6. Chelly J, Concordet JP, Kaplan JC, Kahn A. 1989. Illegitimate transcription: transcription of any gene in any cell type. Proc. Natl. Acad. Sci. U. S. A 86:2617-21.

7. Surget-Groba Y, Montoya-Burgos JI. Optimization of de novo transcriptome assembly from next-generation sequencing data. 2010. Genome Res. 20:1432-40.

8. Mbandi SK, Hesse U, van Heusden P, Christoffels A. 2015. Inferring bona fide transfrags in RNA-Seq derived-transcriptome assemblies of non-model organisms. BMC Bioinformatics 
$16: 58$

9. Bens M, Sahm A, Groth M, Jahn N, Morhart M, Holtze S, et al. 2016. FRAMA: from RNAseq data to annotated mRNA assemblies. BMC Genomics 17:54.

10. He B, Zhao S, Chen Y, Cao Q, Wei C, Cheng X, et al. 2015. Optimal assembly strategies of transcriptome related to ploidies of eukaryotic organisms. BMC Genomics 16:65.

11. Nakasugi K, Crowhurst R, Bally J, Waterhouse P. 2014. Combining Transcriptome Assemblies from Multiple De Novo Assemblers in the Allo-Tetraploid Plant Nicotiana benthamiana. PLoS ONE 9:e91776.

12. Parra G, Bradnam K, Korf I. 2007. CEGMA: a pipeline to accurately annotate core genes in eukaryotic genomes. Bioinformatics 23:1061-7.

13. Simão FA, Waterhouse RM, Ioannidis P, Kriventseva EV, Zdobnov EM. 2015. BUSCO: assessing genome assembly and annotation completeness with single-copy orthologs.

Bioinformatics btv351.

14. Honaas LA, Wafula EK, Wickett NJ, Der JP, Zhang Y, Edger PP, et al. 2016 . Selecting Superior De Novo Transcriptome Assemblies: Lessons Learned by Leveraging the Best Plant Genome. PLoS ONE 11(1):e0146062.

15. Smith-Unna R, Boursnell C, Patro R, Hibberd J, Kelly S. 2016. TransRate: reference free quality assessment of de novo transcriptome assemblies. Genome Res. 26(8):1134-44.

16. Li B, Fillmore N, Bai Y, Collins M, Thomson JA, Stewart R, et al. 2014. Evaluation of de novo transcriptome assemblies from RNA-Seq data. Genome Biol. 15(12):553

17. Davidson NM, Oshlack A. 2014. Corset: enabling differential gene expression analysis for de novo assembled transcriptomes. Genome Biol. 15(7):410

18. Fu L, Niu B, Zhu Z, Wu S, Li W. 2012. CD-HIT: accelerated for clustering the nextgeneration sequencing data. Bioinformatics 28:3150-2.

19. Pertea G, Huang X, Liang F, Antonescu V, Sultana R, Karamycheva S, et al. 2003. TIGR Gene Indices clustering tools (TGICL): a software system for fast clustering of large EST datasets. Bioinformatics 19:651-2.

20. Li B, Dewey CN. 2011. RSEM: accurate transcript quantification from RNA-Seq data with or without a reference genome. BMC Bioinformatics 12:323. 


\section{Figure 1 (on next page)}

Steps in runDRAP workflow

This workflow is used to produce an assembly from one sample/tissue/development stage. It take as input R1 from single-end sequencing or R1 and R2 from paired-end sequencing and eventually a reference proteins set from closest species with known proteins. 


\section{Figure 2 (on next page)}

Steps in runMeta workflow

This workflow is used to produce a merged assembly from several

samples/tissues/development stage outputted by runDRAP. Inputs are runDRAP output folders and eventually a reference protein set. 

Figure 3 (on next page)

Steps in runAssessment workflow

This workflow is used to evaluate quality for one assembly or for compare several assemblies produced from the same dataset. Inputs are the assembly/ies, R1 and eventually R2, and a reference protein set. 


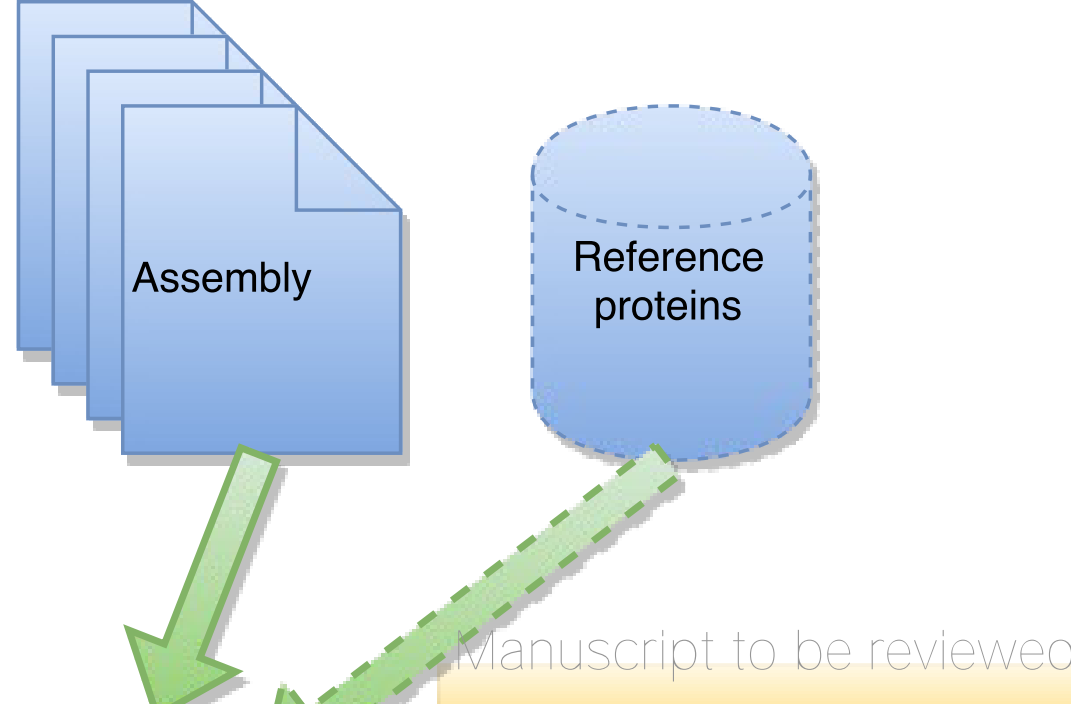

Metrics on length and number of contigs

Number of contigs included in other(s)

- Number of contigs with inner repetition(s)

- Size of inner repetition(s)

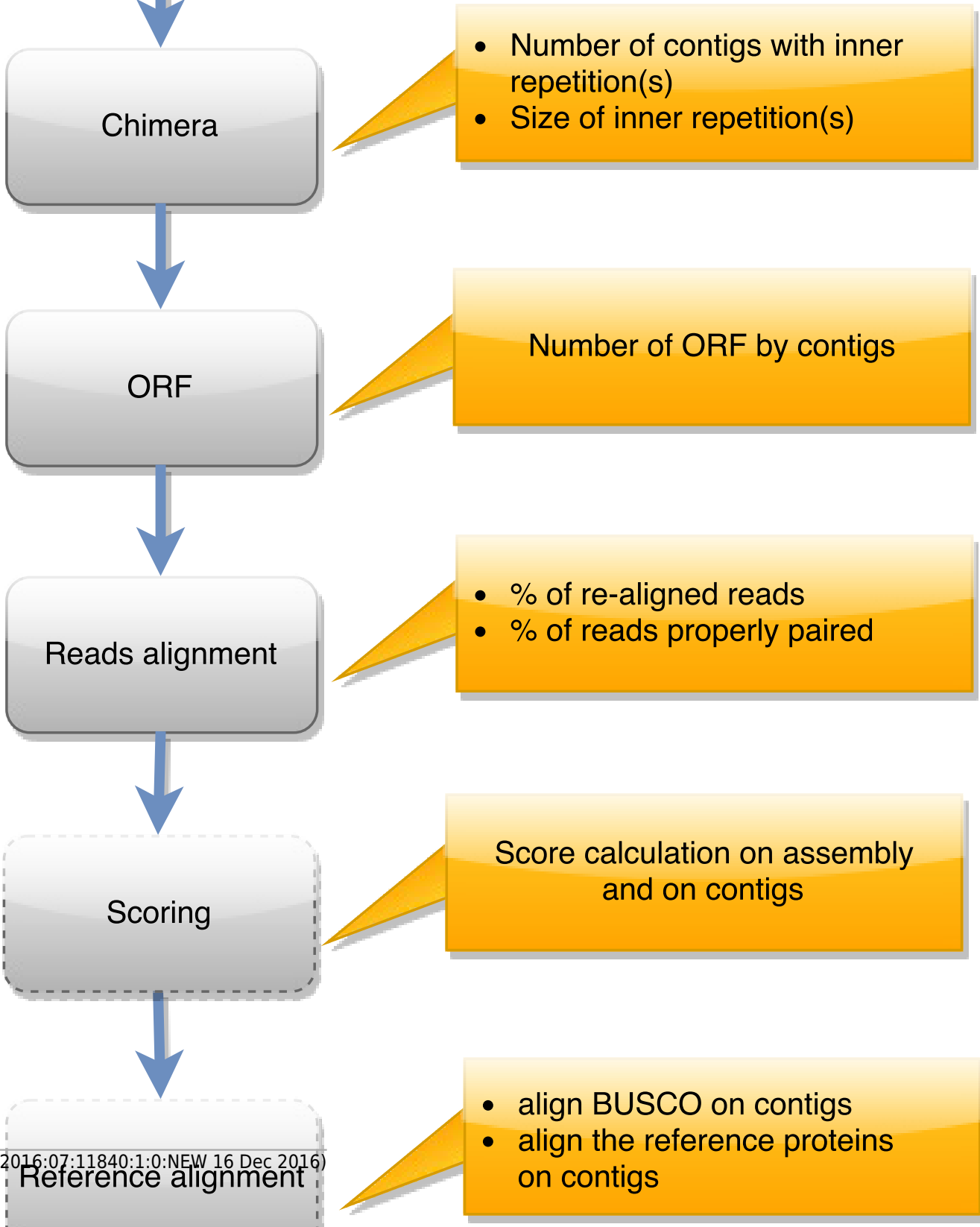

Peer) reviewing PDF | (2016:07:11840:1:0:NEWW 16 Dec 2016)

align the reference protein 


\section{Figure 4 (on next page)}

Number of contigs

The figure shows for the different assemblers (Oases, DRAP Oases, Trinity, DRAP Trinity) the number of contigs produced for each dataset. 
$800 k$

$600 k$
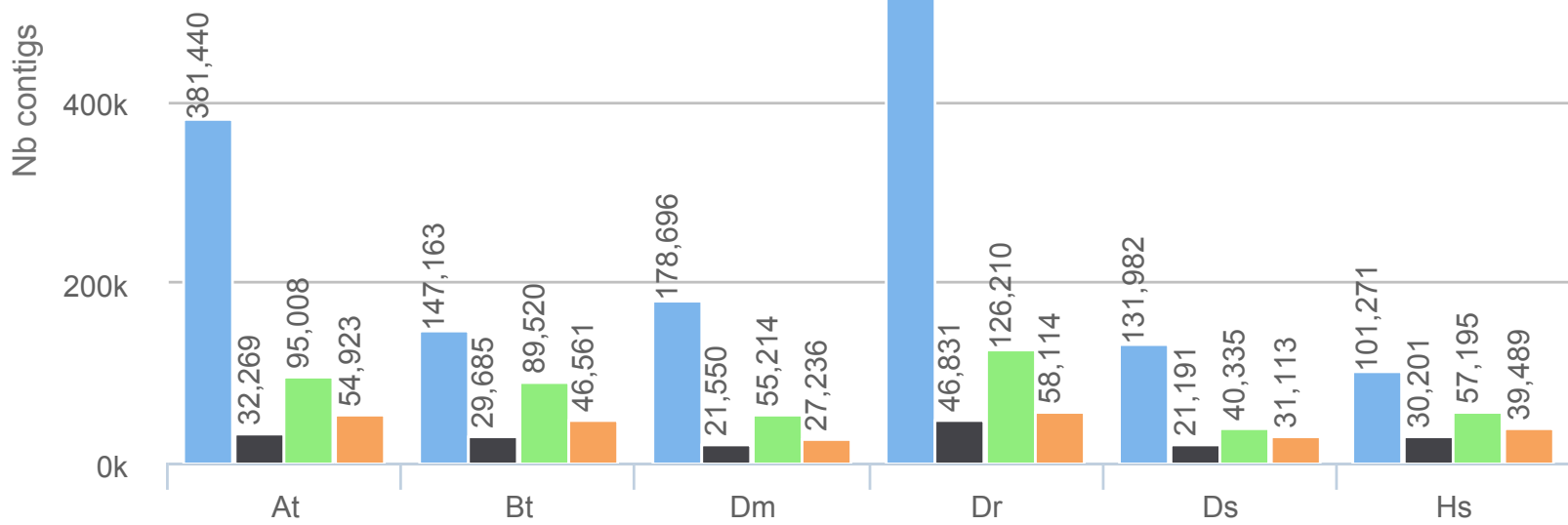
Figure 5 (on next page)

Consensus error rates

Figure A presents the ratio of the global error rates between raw and DRAP assemblies for each dataset (data from table 2 colum 12). Figures B, C and D present the ratio of the error rates respectively for substitution, insertions and deletions between raw and DRAP assemblies for each dataset (data from supplemental table S2). 
(B) Manuscript to bepesensefs substitution rate

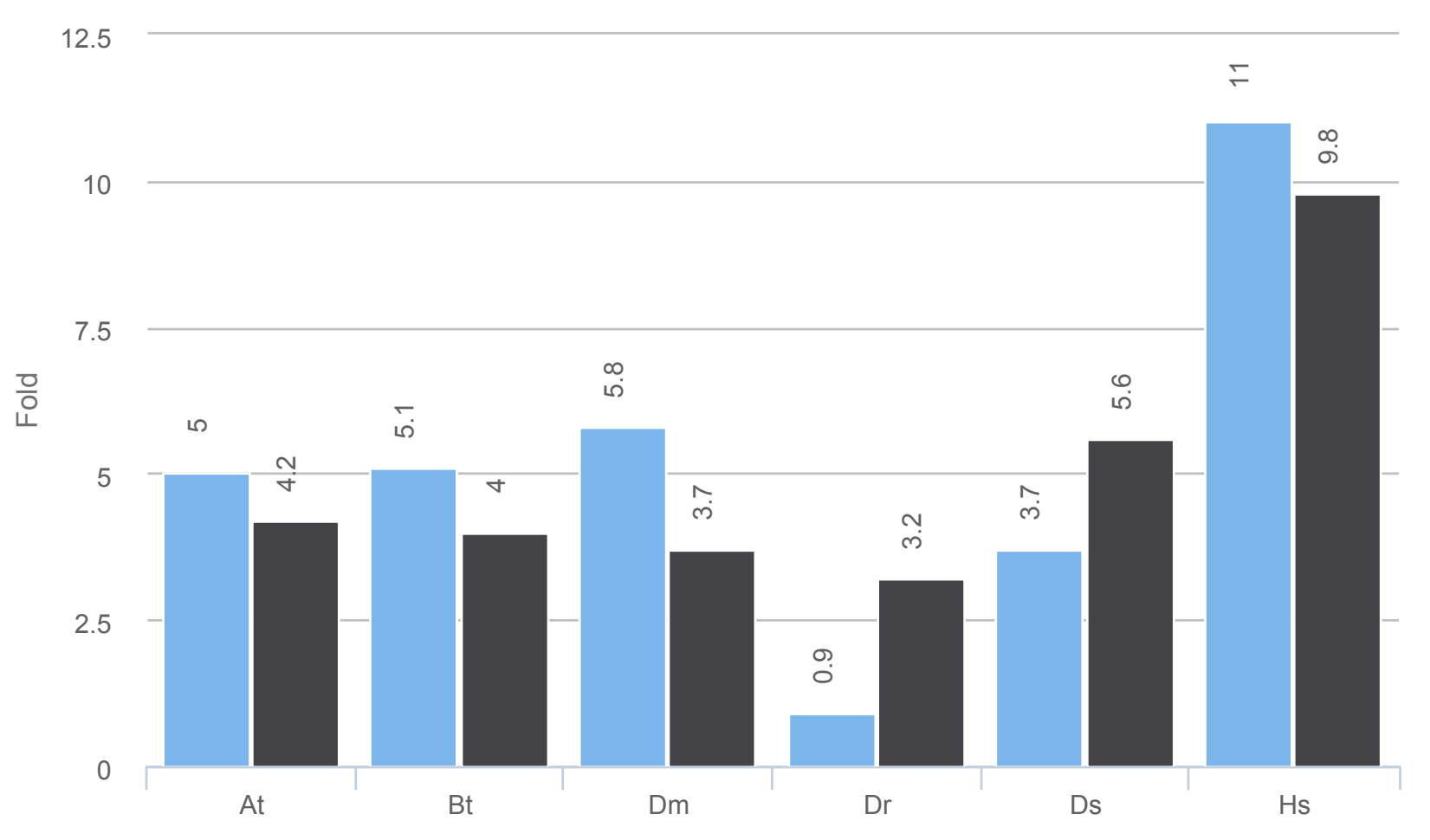

(C)

Consensus insertion rate

(D)

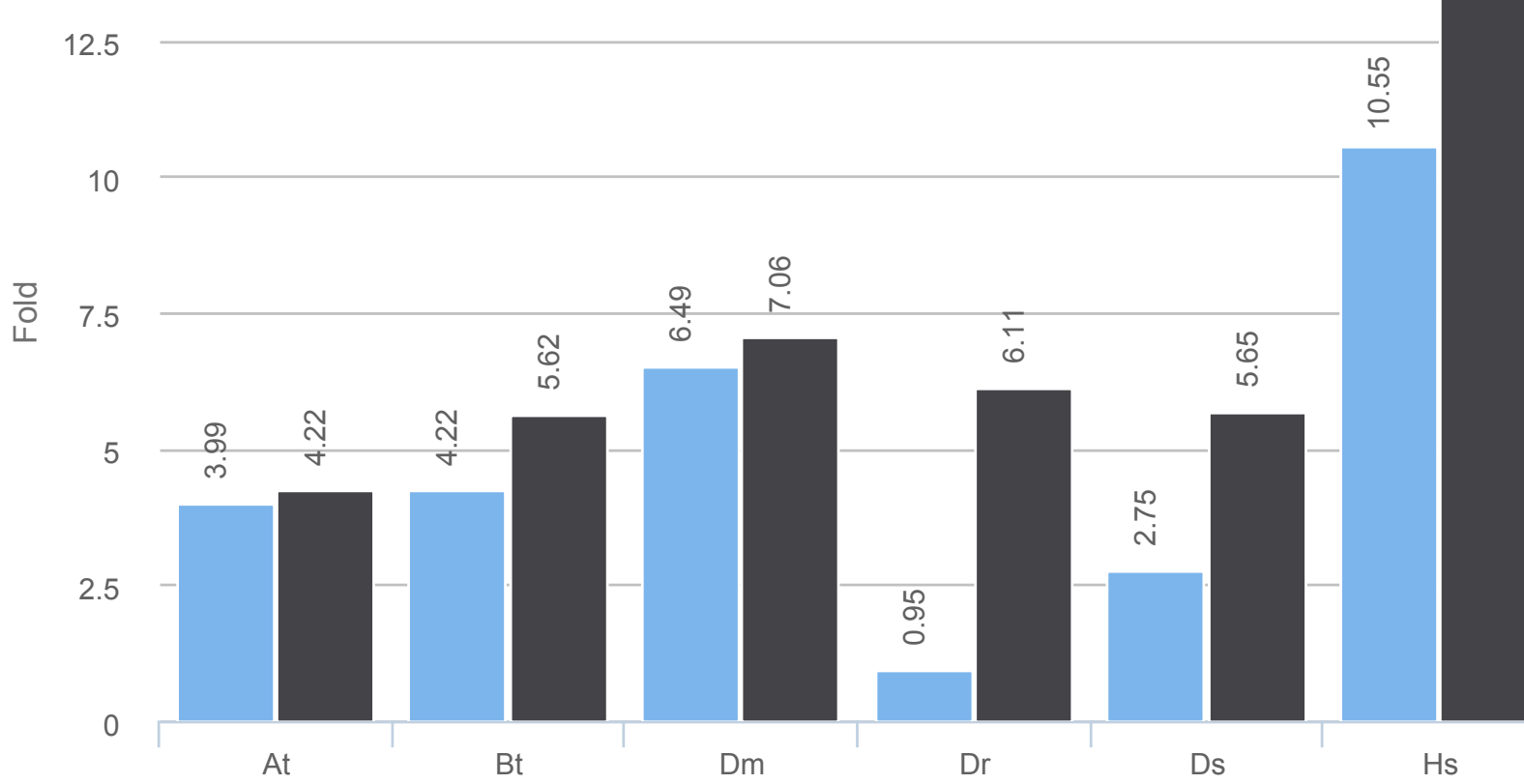

Consensus deletion rate
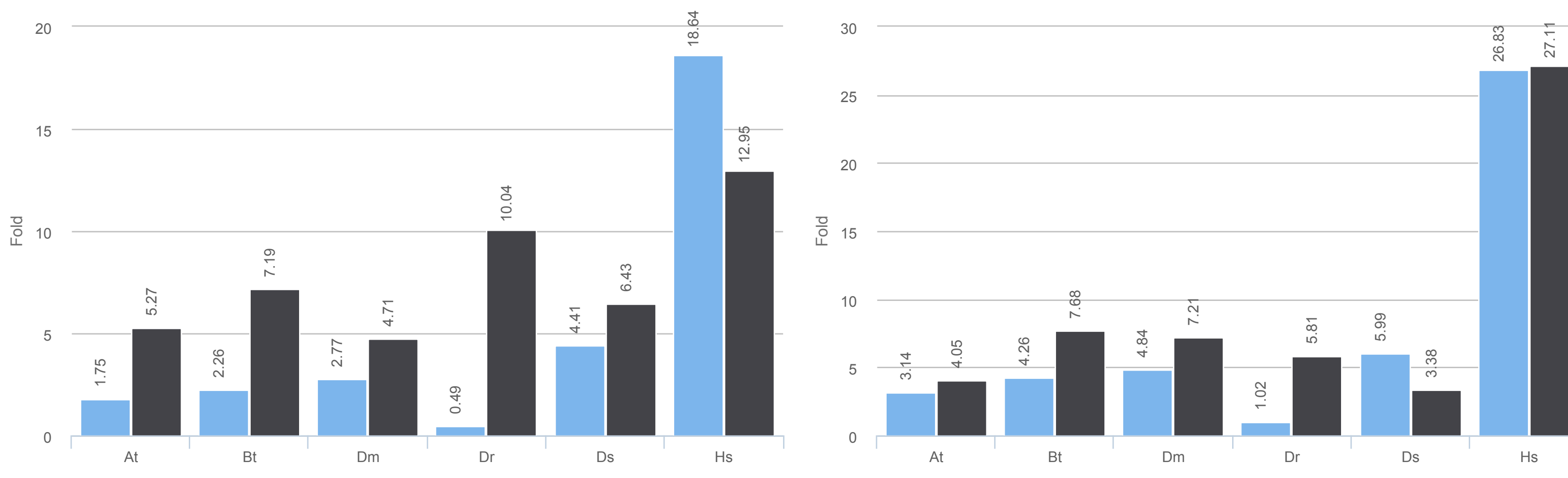

25

20

응 15

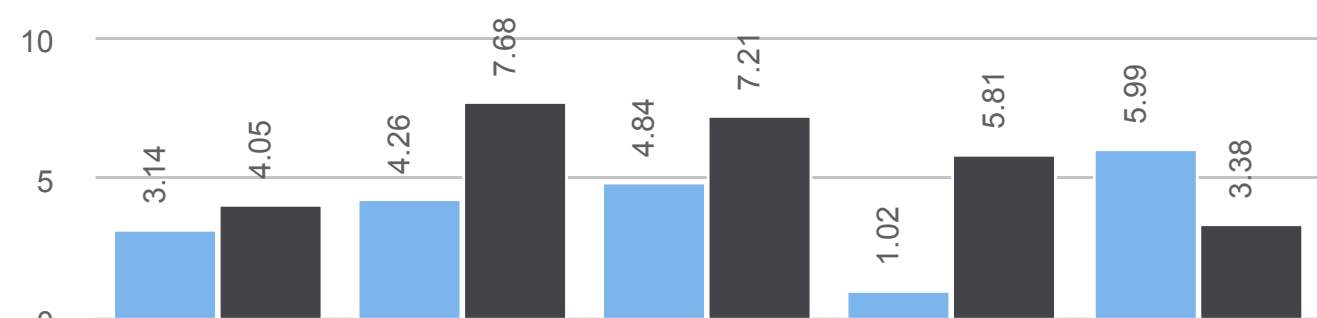

At

$\mathrm{Bt}$

Dm

Dr

Ds

Hs 
Figure $\mathbf{6}$ (on next page)

Reads re-alignment rates

Figures $A$ and $B$ show respectively the alignment rates for reads and read pairs for the four assemblies of each dataset. 
(A) PeerJ

Manuscript to be reviewed

100

సं

75

50

25

0
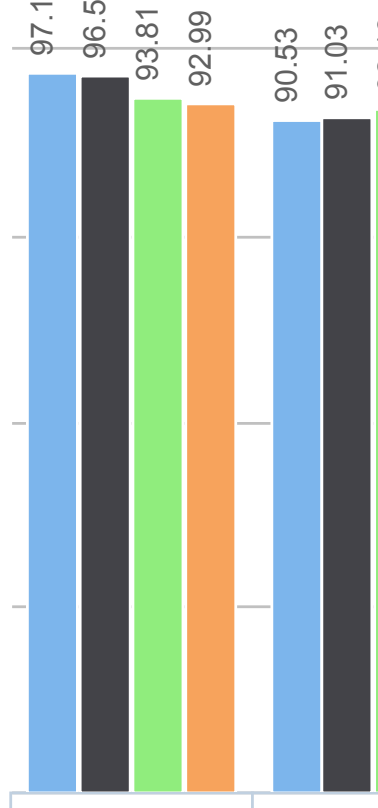

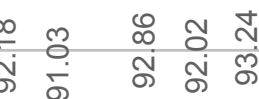

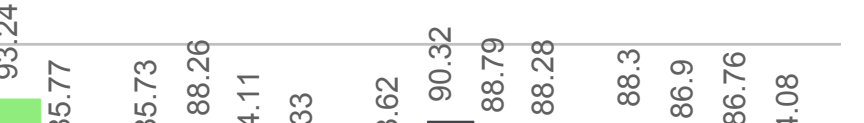

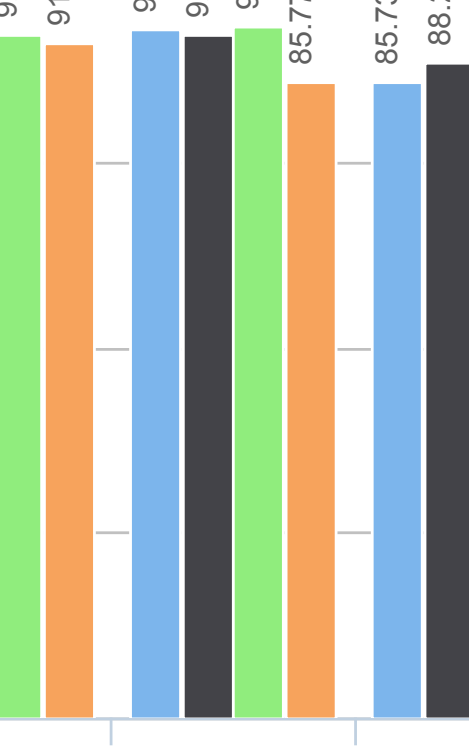

At

Bt

Dm

Dr

Ds

Hs

(B)

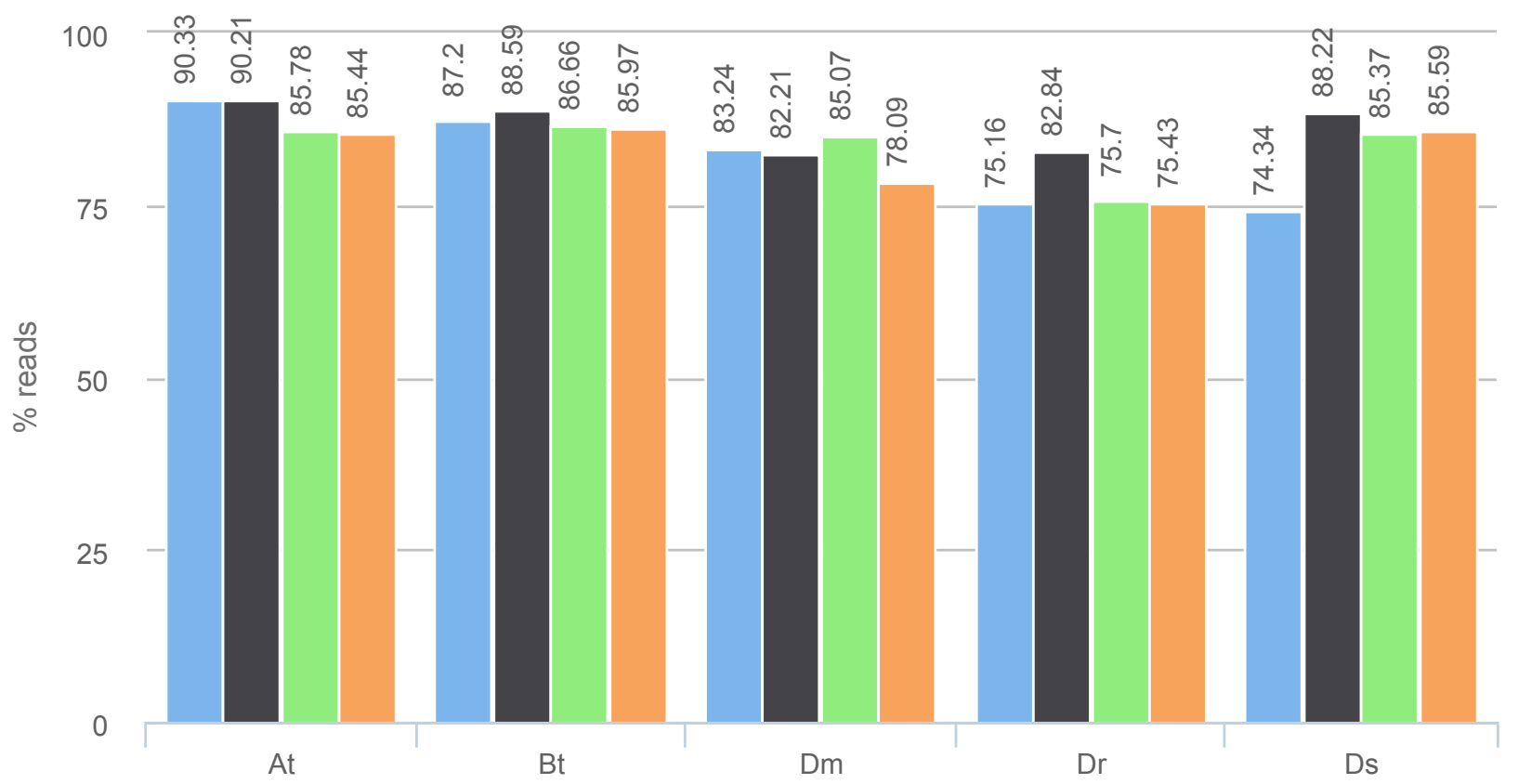


Figure 7 (on next page)

Proteins realignment rates

The figure shows the number of proteins which have been aligned on the contig sets with more than $80 \%$ identity and $80 \%$ coverage for each assembler and dataset. 


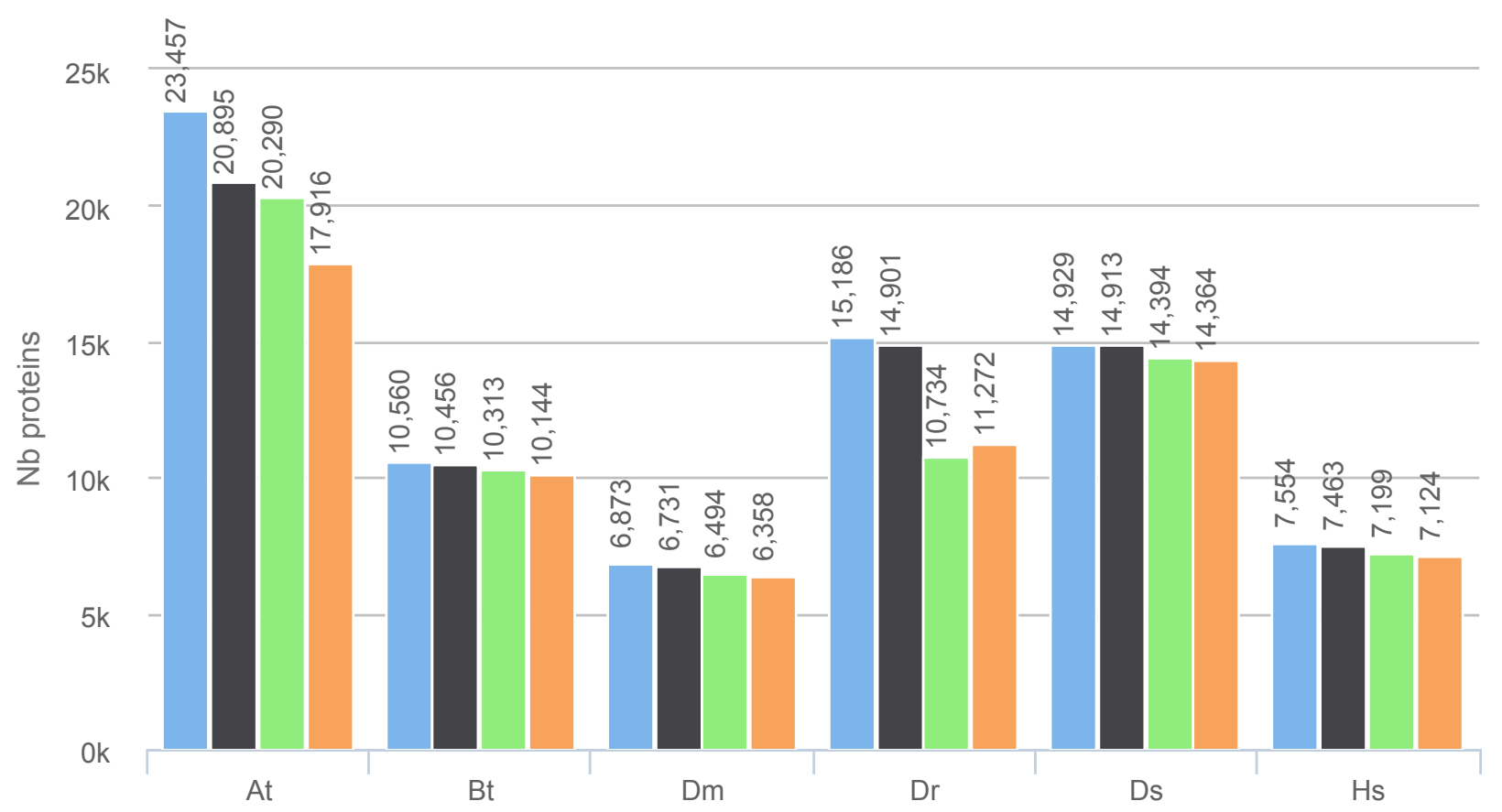




\section{Figure 8 (on next page)}

\section{TransRate scores}

The figure presents the TransRate scores of the four assemblers for each dataset. 
0.8

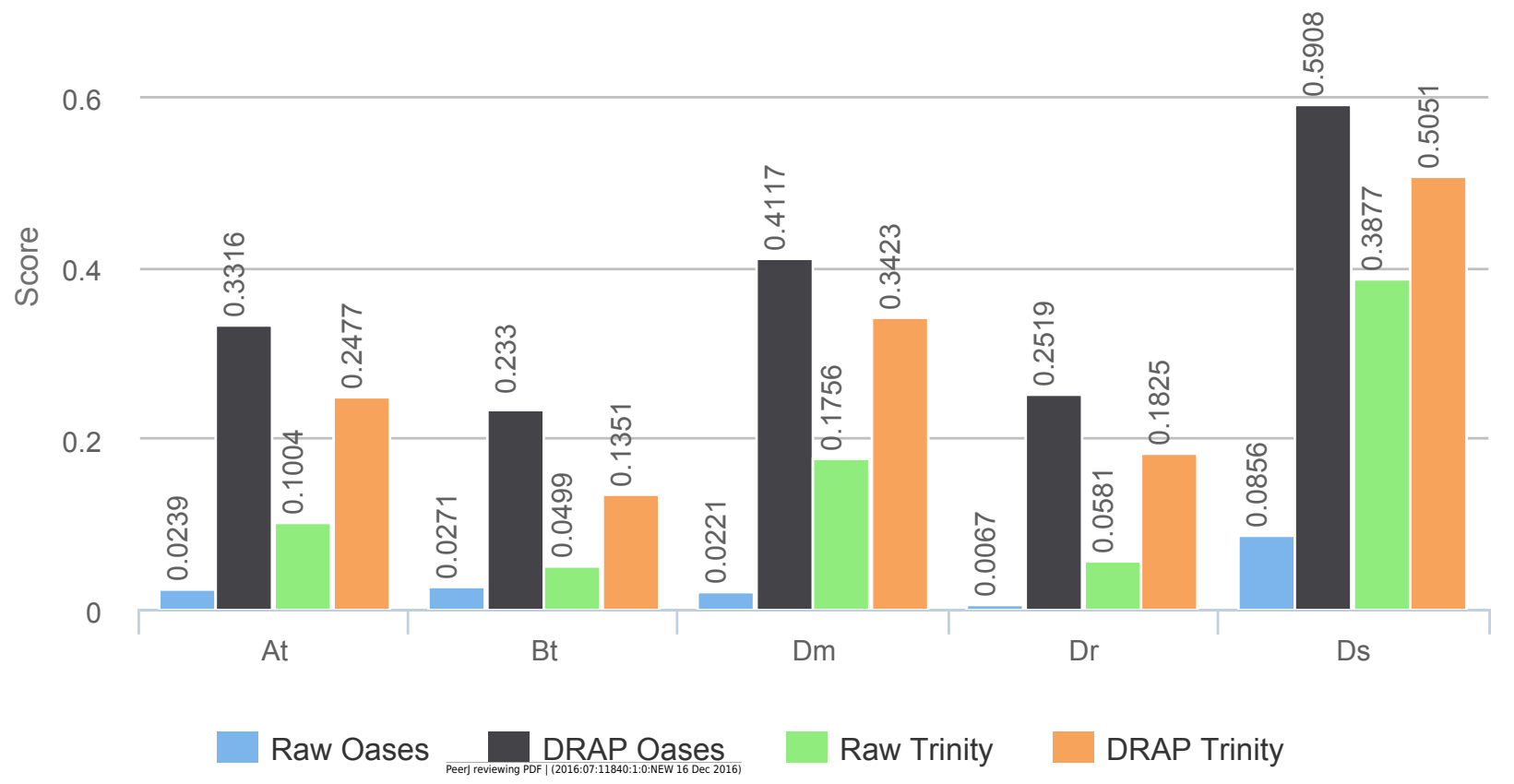


Figure 9 (on next page)

Gene reconstruction versus expression depth using simulated reads

The figure presents the proportion of correctly build transcripts (method presented in Article S1) versus the read count per transcript. 


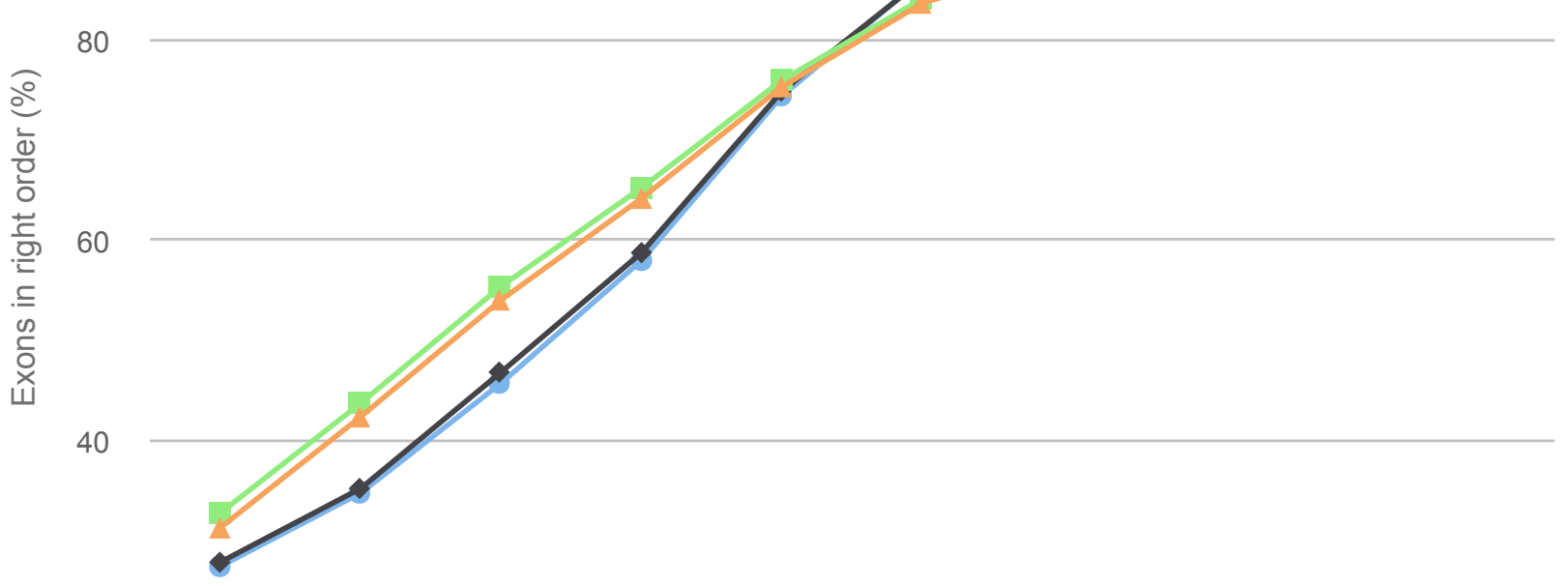

20

$[1 ; 18][19 ; 38] \quad[39 ; 70] \quad[71 ; 123][124 ; 202][203 ; 328][329 ; 523][524 ; 890][891 ; 1784][1785 ; \infty[$ Reads count

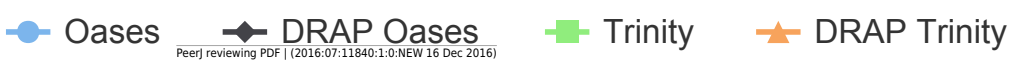




\section{Table 1 (on next page)}

Datasets 


\begin{tabular}{|c|c|c|c|c|c|c|c|c|}
\hline \multirow[b]{2}{*}{ Name } & \multirow[b]{2}{*}{ Species } & \multicolumn{3}{|c|}{ Layout } & \multicolumn{2}{|l|}{ Library } & \multicolumn{2}{|l|}{ Protocol } \\
\hline & & $\begin{array}{l}\text { Paire } \\
\text { d }\end{array}$ & Stranded & $\begin{array}{l}\text { Length } \\
\text { (nt) }\end{array}$ & Nb R1 & SRA ID & Tissue & Condition \\
\hline \multirow[t]{5}{*}{ At } & Arabidopsis thaliana & Yes & - & 100 & 32041730 & SRR1773557 & Root & Full nutrition \\
\hline & & Yes & - & 100 & 30990531 & SRR1773560 & Shoot & Full nutrition \\
\hline & & Yes & - & 100 & 24898527 & SRR1773563 & Root & $\mathrm{N}$ starvation \\
\hline & & Yes & - & 100 & 54344171 & SRR1773569 & Flower & Full nutrition \\
\hline & & Yes & - & 150 & 31467967 & SRR1773580 & Shoot & $\mathrm{N}$ starvation \\
\hline \multirow[t]{3}{*}{$\mathrm{Bt}$} & Bos taurus & Yes & No & 100 & 30140101 & SRR2635009 & Milk & Day 70 with low milk production \\
\hline & & Yes & No & 75 & 15339206 & SRR2659964 & Endometrium & - \\
\hline & & Yes & Yes & 50 & 13542516 & SRR2891058 & Oviduct & - \\
\hline \multirow[t]{12}{*}{ Dd } & Danio rerio & Yes & No & 100 & 35368936 & SRR1524238 & Brain & 5 months female \\
\hline & & & & & 54472116 & SRR1524239 & Gills & 5 months female \\
\hline & & & & & 85672616 & SRR1524240 & Heart & 5 months male and female \\
\hline & & & & & 34032976 & SRR1524241 & Muscle & 5 months female \\
\hline & & & & & 59248034 & SRR1524242 & Liver & 5 months female \\
\hline & & & & & 46371614 & SRR1524243 & Kidney & 5 months male and female \\
\hline & & & & & 96715965 & SRR1524244 & Bones & 5 months female \\
\hline & & & & & 43187341 & SRR1524245 & Intestine & 5 months female \\
\hline & & & & & 55185501 & SRR1524246 & Embryo & 2 days embryo \\
\hline & & & & & 24878233 & SRR1524247 & Unfertilized eggs & 5 months female \\
\hline & & & & & 22026486 & SRR1524248 & Ovary & 5 months female \\
\hline & & & & & 59897686 & SRR1524249 & Testis & 5 months male \\
\hline \multirow[t]{4}{*}{$\mathrm{Dm}$} & Drosophila melanogaster & Yes & Yes & 75 & 21849652 & SRR2496909 & Cell line R4 & Time P17 \\
\hline & & & & & 21864887 & SRR2496910 & Cell line R4 & Time P19 \\
\hline & & & & & 20194362 & SRR2496918 & Cell line R5 & Time P17 \\
\hline & & & & & 22596303 & SRR2496919 & Cell line R5 & Time P19 \\
\hline \multirow[t]{5}{*}{$\mathrm{Dr}$} & Danio rerio & Yes & No & 100 & 5072822 & SRR1048059 & Pineal gland & Light \\
\hline & & & & & 8451113 & SRR1048060 & Pineal gland & Light \\
\hline & & & & & 8753789 & SRR1048061 & Pineal gland & Dark \\
\hline & & & & & 7420748 & SRR1048062 & Pineal gland & Dark \\
\hline & & & & & 9737614 & SRR1048063 & Pineal gland & Dark \\
\hline Ds & Danio rerio & Yes & No & 100 & 30000000 & Simulated & - & - \\
\hline \multirow[t]{4}{*}{$\mathrm{Hs}$} & Homo sapiens & No & No & $25-50$ & 15885224 & SRR2569874 & TK6 cells & pretreated with the protein kinase $\mathrm{C}$ activating tumor \\
\hline & & & & & 15133619 & SRR2569875 & TK6 cells & pretreated with the protein kinase $\mathrm{C}$ activating tumor \\
\hline & & & & & 19312543 & SRR2569877 & TK6 cells & pretreated with the protein kinase $\mathrm{C}$ activating tumor \\
\hline & & & & & 21956840 & SRR2569878 & TK6 cells & pretreated with the protein kinase $\mathrm{C}$ activating tumor \\
\hline
\end{tabular}


Table 2 (on next page)

Compaction and correction in DRAP and standard assembler 


\begin{tabular}{|c|c|c|c|c|c|c|c|c|c|c|c|}
\hline $\begin{array}{l}\text { Datase } \\
t\end{array}$ & Assembler & $\begin{array}{l}\text { Nb } \\
\text { contigs }\end{array}$ & $\begin{array}{l}\text { N50 } \\
\text { (nt) }\end{array}$ & L50 (nt) & $\operatorname{Sum}(n t)$ & $\begin{array}{l}\text { Median } \\
\text { Length } \\
\text { (nt) }\end{array}$ & $\begin{array}{l}\text { Included } \\
\text { Contigs (\%) }\end{array}$ & $\begin{array}{l}\text { Contigs with } \\
\text { Multi-ORF } \\
(\%)\end{array}$ & $\begin{array}{l}\text { Contigs with } \\
\text { Multi-prot (\%) }\end{array}$ & $\begin{array}{l}\text { Chimeric } \\
\text { Contigs (\%) }\end{array}$ & $\begin{array}{l}\text { Contigs with } \\
\operatorname{Bias}^{*}(\%)\end{array}$ \\
\hline \multirow[t]{4}{*}{ At } & Oases & 381440 & 2971 & 92020 & 843329264 & 1816 & 72.75 & 27.89 & 0.26 & 0.80 & 13.88 \\
\hline & DRAP Oases & 32269 & 2014 & 9563 & 56122047 & 1547 & 0.00 & 0.24 & 1.40 & 0.04 & 2.78 \\
\hline & Trinity & 95008 & 2198 & 19140 & 130969737 & 991 & 4.05 & 15.63 & 1.22 & 0.20 & 11.29 \\
\hline & DRAP Trinity & 54923 & 1761 & 15857 & 80258659 & 1287 & 0.00 & 0.20 & 0.52 & 0.00 & 2.68 \\
\hline \multirow[t]{4}{*}{$\mathrm{Bt}$} & Oases & 147163 & 2739 & 31441 & 269085141 & 1359 & 71.19 & 7.45 & 0.06 & 0.66 & 6.29 \\
\hline & DRAP Oases & 29685 & 2441 & 6029 & 47727730 & 1111 & 0.00 & 0.28 & 0.32 & 0.03 & 1.23 \\
\hline & Trinity & 89520 & 2184 & 12080 & 90989611 & 431 & 4.12 & 3.69 & 0.17 & 0.12 & 5.98 \\
\hline & DRAP Trinity & 46561 & 2129 & 9183 & 64809448 & 927 & 0.00 & 0.23 & 0.14 & 0.00 & 1.50 \\
\hline \multirow[t]{4}{*}{$\mathrm{Dm}$} & Oases & 178696 & 2220 & 29086 & 232776717 & 756 & 75.48 & 5.14 & 0.18 & 0.35 & 13.11 \\
\hline & DRAP Oases & 21550 & 2309 & 3674 & 29372261 & 804 & 0.00 & 0.09 & 0.45 & 0.06 & 2.27 \\
\hline & Trinity & 55214 & 2266 & 7126 & 57209890 & 438 & 5.19 & 4.58 & 0.95 & 0.22 & 13.33 \\
\hline & DRAP Trinity & 27236 & 2146 & 5240 & 37249612 & 914 & 0.00 & 0.07 & 0.31 & 0.00 & 3.59 \\
\hline \multirow[t]{4}{*}{$\mathrm{Dr}$} & Oases & 702640 & 2715 & 114042 & 1059904844 & 857 & 70.99 & 2.80 & 0.01 & 1.39 & 11.52 \\
\hline & DRAP Oases & 46831 & 2757 & 9046 & 82268872 & 1173 & 0.00 & 0.15 & 0.27 & 0.16 & 13.05 \\
\hline & Trinity & 126210 & 1279 & 21003 & 96279046 & 418 & 5.56 & 0.81 & 0.08 & 0.56 & 23.63 \\
\hline & DRAP Trinity & 58114 & 1644 & 13022 & 68900396 & 866 & 0.00 & 0.07 & 0.12 & 0.00 & 7.41 \\
\hline \multirow[t]{4}{*}{ Ds } & Oases & 131982 & 2975 & 28618 & 280469694 & 1619 & 75.05 & 3.05 & 0.06 & 0.14 & 4.07 \\
\hline & DRAP Oases & 21191 & 3000 & 4872 & 46994928 & 1744 & 0.00 & 0.08 & 0.25 & 0.02 & 1.10 \\
\hline & Trinity & 40335 & 2398 & 7159 & 58571859 & 910 & 3.12 & 1.82 & 0.37 & 0.09 & 6.47 \\
\hline & DRAP Trinity & 31113 & 2381 & 6492 & 51580407 & 1205 & 0.00 & 0.04 & 0.14 & 0.00 & 1.15 \\
\hline \multirow[t]{4}{*}{$\mathrm{Hs}$} & Oases & 101271 & 2048 & 20131 & 132681065 & 895 & 55.73 & 5.55 & 0.03 & 0.11 & 7.51 \\
\hline & DRAP Oases & 30201 & 1880 & 5542 & 34670862 & 540 & 0.00 & 0.15 & 0.08 & 0.00 & 0.68 \\
\hline & Trinity & 57195 & 1687 & 7843 & 47639190 & 384 & 2.63 & 2.85 & 0.12 & 0.09 & 5.79 \\
\hline & DRAP Trinity & 39489 & 1705 & 6621 & 38557758 & 540 & 0.00 & 0.11 & 0.06 & 0.00 & 0.59 \\
\hline
\end{tabular}

1 (*) contigs with consensus variations corrected by DRAP

2 Bold values are "best in class" values between raw and DRAP assemblies 
Table $\mathbf{3}$ (on next page)

Validation DRAP against standard assembler 


\begin{tabular}{|c|c|c|c|c|c|c|c|c|c|c|}
\hline \multirow{2}{*}{ Dataset } & \multirow{2}{*}{ Assembler } & \multicolumn{2}{|c|}{$\begin{array}{l}\text { \% contigs by } \\
\text { ORF count }\end{array}$} & \multirow[t]{2}{*}{$\begin{array}{l}\text { Contigs with } \\
\text { Complete ORF (\%) }\end{array}$} & \multicolumn{2}{|c|}{$\begin{array}{l}\% \text { contigs by } \\
\text { Proteins count }\end{array}$} & \multirow[t]{2}{*}{$\begin{array}{l}\text { Nb reference } \\
\text { Proteins aligned }\end{array}$} & \multicolumn{2}{|c|}{ Reads mapping (\%) } & \multirow[t]{2}{*}{$\begin{array}{l}\text { TransRate } \\
\text { score *100 }\end{array}$} \\
\hline & & 0 & 1 & & 0 & 1 & & Mapped & Properly paired & \\
\hline \multirow[t]{4}{*}{ At } & Oases & 18.96 & 53.15 & 65.72 & 94.27 & 5.57 & 23457 & 97.18 & 90.33 & 2.39 \\
\hline & DRAP Oases & 9.90 & 89.86 & 72.38 & 39.38 & 59.22 & 20895 & 96.53 & 90.21 & 33.16 \\
\hline & Trinity & 38.97 & 45.40 & 40.32 & 81.09 & 17.69 & 20290 & 93.81 & 85.78 & 10.04 \\
\hline & DRAP Trinity & 13.89 & 85.91 & 55.51 & 69.85 & 29.64 & 17916 & 92.99 & 85.44 & 24.77 \\
\hline \multirow[t]{4}{*}{$\mathrm{Bt}$} & Oases & 36.07 & 56.48 & 28.29 & 93.33 & 6.61 & 10560 & 90.53 & 87.20 & 2.71 \\
\hline & DRAP Oases & 32.59 & 67.13 & 25.70 & 67.63 & 32.05 & 10456 & 91.03 & 88.59 & 23.30 \\
\hline & Trinity & 64.13 & 32.18 & 15.33 & 89.48 & 10.35 & 10313 & 92.18 & 86.66 & 4.99 \\
\hline & DRAP Trinity & 38.55 & 61.23 & 24.86 & 79.95 & 19.91 & 10144 & 91.03 & 85.97 & 13.51 \\
\hline \multirow[t]{4}{*}{$\mathrm{Dm}$} & Oases & 46.19 & 48.67 & 20.27 & 96.43 & 3.39 & 6873 & 92.86 & 83.24 & 2.21 \\
\hline & DRAP Oases & 48.80 & 51.11 & 31.45 & 70.30 & 29.25 & 6731 & 92.02 & 82.21 & 41.17 \\
\hline & Trinity & 67.53 & 27.89 & 18.49 & 89.63 & 9.42 & 6494 & 93.24 & 85.07 & 17.56 \\
\hline & DRAP Trinity & 45.94 & 53.99 & 32.23 & 77.76 & 21.93 & 6358 & 85.77 & 78.09 & 34.23 \\
\hline \multirow[t]{4}{*}{ Dr } & Oases & 56.81 & 40.39 & 23.37 & 97.98 & 2.01 & 15186 & 85.73 & 75.16 & 0.67 \\
\hline & DRAP Oases & 40.20 & 59.65 & 33.43 & 70.89 & 28.84 & 14901 & 88.26 & 82.84 & 25.19 \\
\hline & Trinity & 66.76 & 32.43 & 9.79 & 92.34 & 7.58 & 10734 & 84.11 & 75.70 & 5.81 \\
\hline & DRAP Trinity & 39.74 & 60.19 & 20.16 & 82.44 & 17.44 & 11272 & 81.33 & 75.43 & 18.25 \\
\hline \multirow[t]{4}{*}{ Ds } & Oases & 24.52 & 72.43 & 41.60 & 89.47 & 10.47 & 14929 & 83.62 & 74.34 & 8.56 \\
\hline & DRAP Oases & 12.80 & 87.11 & 53.73 & 35.56 & 64.19 & 14913 & 90.32 & 88.22 & 59.08 \\
\hline & Trinity & 37.72 & 60.46 & 30.29 & 67.37 & 32.26 & 14394 & 88.79 & 85.37 & 38.77 \\
\hline & DRAP Trinity & 22.85 & 77.11 & 37.65 & $\mathbf{5 7 . 5 3}$ & 42.33 & 14364 & 88.28 & 85.59 & 50.51 \\
\hline \multirow[t]{4}{*}{ Hs } & Oases & 44.51 & 49.94 & 21.18 & 93.04 & 6.93 & 7554 & 88.30 & NA & NA \\
\hline & DRAP Oases & 46.95 & 52.91 & 20.06 & 77.28 & 22.64 & 7463 & 86.90 & NA & NA \\
\hline & Trinity & 69.02 & 28.13 & 11.70 & 88.53 & 11.35 & 7199 & 86.76 & NA & NA \\
\hline & DRAP Trinity & 55.48 & 44.41 & 16.07 & 83.46 & 16.48 & 7124 & 84.08 & NA & NA \\
\hline
\end{tabular}

1 Bold values are "best in class" values between raw and DRAP assemblies 


\section{Table 4(on next page)}

Pooled samples vs meta-assembly strategies on the Danio rerio multi samples dataset (Dd)) 


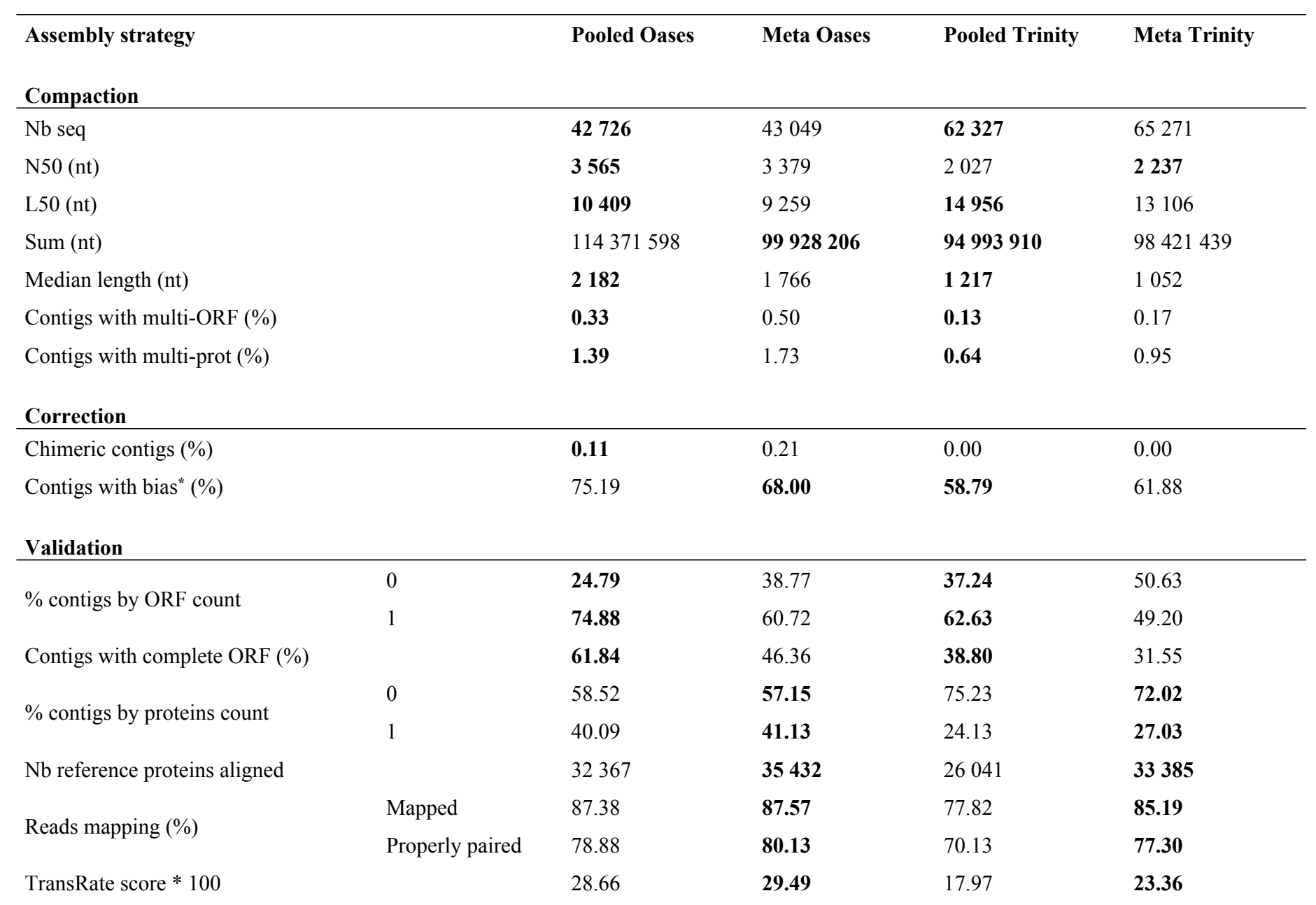

1 (*) contigs with consensus variations corrected by DRAP

2 Bold values are "best in class" values between raw and DRAP assemblies 


\section{Table 5 (on next page)}

Compaction vs gene representation on Danio rerio simulated dataset (Ds) and Danio rerio dataset $(\mathrm{Dr})$ 


\begin{tabular}{|c|c|c|c|c|c|c|}
\hline \multirow{2}{*}{ Dataset } & \multirow{2}{*}{ Assembly } & \multirow{2}{*}{ Nb seq } & \multirow{2}{*}{ All genes } & \multirow{2}{*}{$\begin{array}{l}\text { Multi-isoform } \\
\text { genes }\end{array}$} & \multicolumn{2}{|c|}{ Raw / DRAP assemblies } \\
\hline & & & & & All genes (\%) & Multi-isoform genes (\%) \\
\hline \multirow[t]{4}{*}{ Ds } & Raw Oases & 131982 & 14396 & 3593 & \multirow{2}{*}{-1.74} & \multirow{2}{*}{-82.99} \\
\hline & DRAP Oases & 21191 & 14145 & 611 & & \\
\hline & Raw Trinity & 40335 & 12457 & 1792 & \multirow{2}{*}{-2.04} & \multirow{2}{*}{-17.97} \\
\hline & DRAP Trinity & 31113 & 12203 & 1470 & & \\
\hline \multirow[t]{4}{*}{ Dr } & Raw Oases & 702640 & 11613 & 2177 & \multirow{2}{*}{+10.40} & \multirow{2}{*}{-69.09} \\
\hline & DRAP Oases & 46831 & 12821 & 673 & & \\
\hline & Raw Trinity & 126210 & 8310 & 801 & \multirow{2}{*}{-2.33} & \multirow{2}{*}{-22.60} \\
\hline & DRAP Trinity & 58114 & 8116 & 620 & & \\
\hline
\end{tabular}

1 Bold values are "best in class" values between raw and DRAP assemblies 
Table 6(on next page)

Structure validation on Danio rerio simulated dataset (Ds) 


\begin{tabular}{llllll}
\hline Assembly & $\begin{array}{l}\text { Retrieved } \\
\text { Exons }\end{array}$ & $\begin{array}{l}\text { Exons in } \\
\text { Right contig }\end{array}$ & $\begin{array}{l}\text { Exons in } \\
\text { Right order }\end{array}$ & $\begin{array}{l}\text { Contigs with } \\
\text { More than 1 gene }\end{array}$ & $\begin{array}{l}\text { Max number } \\
\text { Of genes by contig }\end{array}$ \\
\hline Real assembly & $99.81 \%$ & $99.81 \%$ & $99.50 \%$ & $0.16 \%(46)$ & 5 \\
\hline Raw Oases & $80.03 \%$ & $\mathbf{7 7 . 8 3 \%}$ & $\mathbf{7 7 . 6 1 \%}$ & $\mathbf{2 . 7 7 \% ( 5 3 7 )}$ & 221 \\
DRAP Oases & $\mathbf{8 0 . 2 1 \%}$ & $77.54 \%$ & $77.29 \%$ & $4.13 \%(671)$ & $\mathbf{2 0 3}$ \\
Raw Trinity & $\mathbf{8 4 . 2 4 \%}$ & $\mathbf{7 7 . 3 0} \%$ & $\mathbf{7 7 . 1 0} \%$ & $3.65 \%(717)$ & 339 \\
DRAP Trinity & $83.30 \%$ & $76.65 \%$ & $76.47 \%$ & $\mathbf{3 . 1 7} \%(\mathbf{6 0 2})$ & $\mathbf{3 2 7}$
\end{tabular}

1 Bold values are "best in class" values between raw and DRAP assemblies 